\title{
Cloud Computing Intelligent Management by Metaheuristic Algorithm of Intelligent Water Drop
}

\author{
Majid Abdous ${ }^{1}$, Touraj Banirostam ${ }^{2}$ \\ Master Student, Department of Computer Engineering, Electronic Branch, Islamic Azad University, Tehran, Iran ${ }^{1}$ \\ Assistant Professor, Dept. of Computer Engineering, Islamic Azad University, Central Tehran Branch, Tehran, Iran ${ }^{2}$
}

\begin{abstract}
One of the most important challenges in Cloud Network is the matter of allocating physical resources to virtual machines. The goal of this paper is to present a method to have an optimized installation of virtual machines' resources onto physical machines with intelligent water drop so that overall energy consumption in data center is reduced with decreasing the number of active physical servers. Utilizing policies to host overload detection and immigrant virtual machine; and through comparing results of simulation with existing methods; is the best method to manage physical resources in cloud networks which for this purpose, intelligent water drop algorithm has been used. Also to simulate cloud environment, Cloudsim 3.0 simulator library in Netbeans IDE 7.4 develop environment are going to be used. Results shows that in comparison with other methods, the algorithm of intelligent water drop using MAD algorithms as host overload detection algorithm and MMT as the algorithm of selecting virtual machine, the most optimized answer will be found for physical resource management in cloud computing.
\end{abstract}

Keywords: cloud computing, resource management, water drop algorithm, intelligent water drop.

\section{INTRODUCTION}

Cloud computing has been introduced following other former styles with the goal of providing computational resources, storage and fast and secure communication. Also many services have been offered in cloud computing model. One of service provider models in cloud computing is infrastructure as service which in processing resources (network, service providers, and etc.) have been presented as services. The main base of infrastructure as a service is made up of virtualization technology and this mechanism brings so many benefits for cloud network in addition to providing the possibility of integrating independent physical resources in form of virtual resources. In infrastructure as a service, the smallest mutual part between customer and service providers is virtual machine. Request for virtual resources is cited as parameters like CPU speed, memory capacity and disc volume and received requests are provided through mapping virtual resources on physical resources [1].

Moreover, a cloud system is considered to be appropriate for a vast range of users because using that does not require being familiar with infrastructural systems and mastering them. Therefore, anyone can use its service providers just as they use urban power grid regardless to the existing complexity [1].

One another highly important cited challenges in infrastructure as a service is management and allocation of resources to the virtual machines. The main problem of resource allocation in infrastructure as a service is receipting new request and locating that on the virtual machine. Assuming $\mathrm{n}$ virtual machine and $\mathrm{m}$ physical machine, this locating will have $m^{n}$ different ways which gives a large probability space so that searching in such big space and finding a proper answer will be highly timeconsuming. On the other hand, customers expect cloud service providers to do QoS controls on guest virtual machines. Thus, resource management in cloud computing bounds infrastructure management to present a series of purposeful controls in order to balance the quality of guests' services with the efficiency of the whole data center's resources. The problem of optimized locating of virtual service providers on physical machines installed in data center is similar to the problem of packing boxes which is considered to be in the class of "Hard NP" optimization problems in terms of computational complexity [2]. But anyways, optimized solution has been presented fo so many samples of this problem using complex algorithms which are going to be surveyed in literature review section.

Among these complexities, furthermore, a method of optimization and guidance is required to provide a more optimized way. One of these solutions would be using metaheuristic algorithms; choosing a proper algorithm to manage cloud computing resources will be one of the most important matters of discussion in this research. Heuristic has a subpart called metaheuristic which has natureinspired algorithms to solve complex problems. One of these algorithms is intelligent water drop which will be used for simulation in current paper regarding its favorable optimization in other similar problems.

Basically water drop algorithm can be used for both combinatorial and continuous optimization. Water drop is composed of some intelligent water drops which are in 


\section{International Journal of Advanced Research in Computer and Communication Engineering}

Vol. 5, Issue 3, March 2016

both competence and cooperation with each other to find In a paper, PALANIKKUMAR et al, [6] has been better solutions and by changing soils of the graph paths to surveying "An Intelligent Water Drops Algorithm Based better solutions become more reachable [2].. Considering Service Selection and Composition in Service Oriented mentioned matters, it is tried in this paper to use IWD Architecture". Some ideas have been embedded in website algorithm in order to reach a method of optimal through selection of service web and its composition to installment of VMs on physical machines installed in data provide qualified and efficient service in mentioned paper. center with the goal of decreasing power consumption and The proposed algorithm shows a better performance in consequently decreasing both the number of active physical servers and volume of waste in resources.

computation and optimization of Particle Swarm Algorithm (PSO).

Continuing the discussion, history of research will be first Lin et al, [7] have used an algorithm composed of ant surveyed. After that in the third section, research colony and colonial to plan cloud network resources. methodology and mapping method of IWD algorithm in Regarding to the features of ant colony algorithm beside order to manage cloud computing resources will be colonial (CSA), they tried to optimize cloud computing described. Afterwards, simulation and results will be resources which the experimental results of this paper presented in the fourth section.

\section{RELATED WORK}

Belglazov et al, [3] proposed a framework to manage resource allocation in cloud computing data center which includes four main parts (customer, interface, green allocator, and virtual machines) with the ultimate goal of decreasing energy consumption, more commitment to service-level agreement, and decreasing the number of immigrants. They treated the problem of resource allocation in a two-stage way. Anyways, the authors of this paper believes that regarding to specific features of cloud computing environment such as being dynamic, scalable, and distributed; algorithms requiring complex computation and machine learning are not quite suitable and there should be algorithms presented to be scalable and/or high level of fault tolerance.

Zho et al, [4] surveyed the strategy of resource planning in cloud computing using ant colony optimization algorithm. The main goal of this paper is to offer a new business in cloud computing and considering that resource planning strategy has key technologies in cloud computing; thus planning tasks and allocating resources optimize the process of cloud computing system structure, performance status, and problems based on that through using ant colony. Results of this research shows that ant colony algorithms has a better performance in optimizing resources in cloud computing.

Lenin \& Surya [5] have surveyed "IWD algorithm to solve reactive power dispatch problem". In this paper, an algorithm has been used to solve sending power problem in a multi-objective power system and modal analysis of system to evaluate static voltage stability. The main objective is to minimize voltage. Generator terminal voltages, reactive power generation of the capacitor banks and tap changing transformer setting are taken as the optimization variables. IWD algorithm has been used in this paper to solve this combinatorial optimization problem. IWD has been inspired by nature and natural rivers. Since natural rivers often find the best path among so many through a swarm-based optimization, this algorithm is probable to find an optimized path from source to destination. This algorithm has been embedded to solve reactive dispatch problem. effective method to improve resource usage in cloud environment.

Basem et al, [8] have studied "Presenting a method to modified IWD its application to optimization problems" in their paper. In order to the efficiency of selection method in construction stage, a solution of IWD has been presented in mentioned paper. To fit this selection method with IWD algorithm, two method of linear ranking and exponential ranking have been proposed. Both rankingbased selection methods aim to solve the identified limitations of the fitness proportionate selection method as well as to enable the IWD algorithm for local optimization and ensure its search diversity.

To evaluate the usefulness of the proposed ranking-based selection methods, a series of experiments pertaining to three combinatorial optimization problems, i.e., rough set feature subset selection, multiple knapsack and travelling salesman problems, is conducted. The results demonstrate that the exponential ranking selection method is able to preserve the search diversity, therefore improving the performance of the IWD algorithm

\section{III.RESEARCH METHOD}

The main challenge of the research is resource management in physical machines so that resources -after completion of the first machine or lack of sufficient spacego to the next alternative which is so much alike with water drop performance i.e., water drops also respectively fill existing spaces in their flow path and continue their path with such an approach and to remember, the goal of this research is finding the most suitable physical machine to install virtual machine onto with exactly the same approach as well.

So it is expected to have a better performance comparing with other previous methods. Therefore, considering library studies and mentioned above discussions in this paper, it is tried to present a method of resource optimization for virtual service providers on physical machines installed in data center. IWD algorithm is used to overcome complexity, organize stored data, and to decrease energy consumption in current servers. The purpose of proposing this algorithm is to decrease very high costs of service providers of infrastructure as a 


\section{International Journal of Advanced Research in Computer and Communication Engineering}

Vol. 5, Issue 3, March 2016

service which is spent for power consumption cost; as well be two policies in order to VM selection for migration in as service providers reach highest benefits through this research which is described in 3-4 section. After resource allocation which is in direct relationship with identifying migration time and selecting VMs to migrate, energy consumption amount. Regarding to aforementioned destination should be specified for every one of VM matters, IWD algorithm can be considered as a useful candidate for migration. Algorithms used for migrate VM method to decrease power consumption and consequently decreasing the number of active physical servers as well as decreasing resource waste.

\section{A. Intelligent Water Drops Algorithm or IWD}

IWD algorithm is a nature-based algorithm working in group. Intrinsically, the IWD algorithm can be used for combinatorial optimization. However, it may be adapted for continuous optimization too. The IWD algorithm has two types of parameters: Static and Dynamic parameters. Static parameters are constant during the process of the IWD algorithm. Dynamic parameters are reinitialized after each iteration of the IWD algorithm.

\section{B. Mapping virtual machine allocation onto physical hosts with IWD algorithm}

Within the challenge of, physical service providers are divided into three subproblems (Anton, 2013). The first one answers the question of "when should a virtual machine migrate?' The second, to 'which machine should migrate?' And the thirds replies to subproblem of 'where virtual machines migrate to?' During a cloud data center's lifetime, it is repetitively required to transfer virtual machines from one physical host to another physical host; which is the matter of discussion in the subproblem of the time of these migration. After identifying migration time in the first subproblem, it should be decided that which one of VMs are suitable to be transferred to a new physical machine which will be discussed in this subproblem. Identifying new and proper location for VMs or in another word, identifying suitable physical hosts for each of VMs is the challenge of the third subproblem. The problem of VM allocation to physical hosts -the third subproblem- is an "np hard" problem.

There are two states which in VMs should migrate: the first is when processing load of a host is so decreased that all its processing power which is it VMs can be transferred to another working physical host and power off that host. In a simpler word, transference happens whenever processing load of a physical host is overly few. The second state which in VM should be decided to migrate is when; in contrary with the first state, processing load of a physical host is excessively high or in another word, the number of VMs on noticed physical host is so high that it is likely to violate SLA contracts because of lack of those physical host resources.

After identifying migration time, if physical host was determined to be underloaded, then all VMs should be migrated to power off that physical host. While if a physical machine's overhead was overloaded, then it should be identified that which VMs should be migrated from mentioned physical host. When overhead of physical machine is determined to be overloaded, Minimum Migration Mime (MMT) and Random Selection (RS) will selection are called VM Placement algorithms. The large number of physical hosts and VMs have resulted in using evolutionary algorithms for problem of VM placement onto physical hosts. Some samples of utilizing these algorithms to solve resource management in cloud computing have been presented in the history of research. Also a new method to solve mentioned problem is going to be cited based on IWD algorithm.

To solve the problem of VM allocation to physical hosts by using IWD algorithm, first the problem should be simulated as a drop. It should be considered for this purpose that there are a number of physical machines and VMs which location should be identified for each VM so that count of physical hosts would be minimum. Actually there are $\mathrm{N}$ virtual machine selected for migration and their destination is identified. The new location of VM also should be specified in every answers achieved by IWD algorithm in order to give an appropriate answer for computation. For this purpose, every answers should be considered as an array with the length of $\mathrm{N}$ which the amount of each indices represents the number of destination physical host. In table 1, drops are coded for this problem so that the first row shows destination index in physical machine and the second row represents indices of VM arrays. As demonstrated in table 1, VM 0 and 3 should be allocated to physical host 4 . After coding drops, the process of standard IWD which has been previously described, continues.

Table 1 . The way of coding drop in proposed method

\begin{tabular}{|c|c|c|c|c|c|}
\hline value & 4 & 2 & 3 & 4 & 1 \\
\hline index & 0 & 1 & 2 & 3 & 4 \\
\hline
\end{tabular}

1- Service Level Agreement (SLA)

Service Level Agreement (SLA) is the agreement on quality level of service or service quality guarantee. It is an official contract between service providers and service users concluded in order to assure quality parameters of service. SLM is one of applicable methods of IT and Telecom management which was presented in the second half of 1990s to build a win/win relationship between two participants involved in ICT services I.e., service providers and service users (Microsoft, 2003)

\section{2- Host overload and underload detection algorithm}

"One-Threshold Algorithm" can be mentioned as one of well-known host underload detection algorithm and "Local Regression Algorithm, Local Regression Robust Algorithm, and Interquartile Range algorithm, and the Median Absolute Deviation algorithm" can be cited as host overload detection algorithms. So it can be said that well-known detection algorithms are as below:

- One-Threshold Algorithm: This algorithm is applicable when physical host is underloaded since in this situation, host is allocating $70 \%$ of its consuming 


\section{International Journal of Advanced Research in Computer and Communication Engineering}

Vol. 5, Issue 3, March 2016

energy to itself, this algorithm makes decision through considering one host as criteria to find if system is underload or working to continue activities or migrating virtual machine and powering off the system.

- Local Regression Algorithm: LR algorithm is based on leoess proposed by Cliveland in 1992. The main foundation of this method is simple connection models for local data subsets in order to build an approximate curve for main data.

- Median Absolute Deviation algorithm: MAD is a strong statistical analysis which has the most flexibility to remote nodes in a complex in proportion to the standard deviation (Beloglazov et at, 2012)

- Local Robust Regression Algorithm: aforementioned loess version is vulnerable to remote nodes which can be generated by heavy-tailed or leptokurtic distribution (Gunz e al., 2011)

- Interquartile Range Algorithm: In descriptive statistics, IQR is also called midspread or middle fifty a measure of statistical dispersion (Beloglazov et al, 201).

\section{Which VM should migrate?}

After determining migration time, if the host was found to be underloaded, all VMs should migrate to another physical host and switch mentioned host to the sleep mode while if the host overloading happened, the second subproblem will come up to be to determine which VM should be migrated from noticed physical host. Two dominant policies used in this paper to select VMs from sender hosts in state of being overused are (Beloglazov \& Buyya, 2012):

- The Minimum Migration Time Policy (MMT): selecting VMs requiring the minimum time to complete a migration in order to minimize migration overhead since VM with smaller Memory and CPU can migrate faster, actually small VMs are picked for migration in this method, thus, this policy consequently leads this method to have a good performance if there was no need for a large Memory or CPU free up to go out of overloading status. But otherwise, i.e., if going out of overloading status required large free up, a great number of VM s should be migrated that in this case, MMT method will not have a good performance (Beloglazov \& Buyya, 2012).

- Random Selection (RS): RS is migration of required number of VMs packed according to a uniformly distributed discrete random variable. This method; or in another word, general cloud computing center; can be considered as an appropriate approach for data centers with large number of VMs (Buyya et al, 2010).

\section{Calculating Fitness Functionality}

To identify how much a solution can present an appropriate answer, its value should be measured by fitness functionality. According to effective parameters in quality of solution, this functionality relates a value to that. By applying this functionality on all solutions in proposed algorithm, their values will be separately calculated and the one with the best value will be considered as the most appropriate solution. In modeling this problem, parameters have been defined as below:

$\mathrm{V}$ : Collection of VMs

P: Collection of Physical hosts

$\mathrm{V}_{\mathrm{i}}$ : One $\mathrm{VM}$ in $\mathrm{V}$

$\mathrm{V}_{\mathrm{i}} \mathrm{Cpu}$ : Required Amount of CPU for $\mathrm{V}_{\mathrm{i}}$

$\mathrm{V}_{\mathrm{i}}$ mem: Required Amount of Main Memory for $\mathrm{V}_{\mathrm{i}}$

$\mathrm{P}_{\mathrm{j}}$ : One physical host in $\mathrm{P}$

$\mathrm{P}_{\mathrm{j}} \mathrm{Cpu}$ : Required Amount of CPU for $\mathrm{P}_{\mathrm{j}}$

Pjmem: Required Amount of Main Memory for $\mathrm{P}_{j}$

$\mathrm{P}_{\mathrm{j}} w \mathrm{cpu}$ : Total working load of $\mathrm{p}_{\mathrm{j}}$ 's CPU

$\mathrm{P}_{\mathrm{j}} \mathrm{wmem}$ : Total working load of $\mathrm{p}_{\mathrm{j}}$ 's main memory

$\mathrm{V}_{\mathrm{pj}}$ : The collection of allocated VMs to physical hosts

CPU efficiency rate in $\mathrm{P}_{\mathrm{j}}$ physical host is based on Relationship (1):

$$
\text { (1) } \mu j=P_{j}^{w_{c p u}} / P_{j}^{c p u}
$$

So energy consumption is calculated from relationship (2):

$$
\text { (2) } E\left(p_{j}\right)=k_{j} \cdot e_{j}^{\max }+\left(1-k_{j}\right) \cdot e_{j}^{\max } \cdot \mu_{j}
$$

In relationship (2), $\mathrm{k}_{\mathrm{j}}$ is equal to energy consumption of physical host while being idle, $e_{i}{ }^{\max }$ is energy consumption of physical host $P_{j}$ in the peak of processing load and $M_{j}$ shows CPU efficiency amount of $\mathrm{P}_{\mathrm{j}}$. Therefore, fitness functionality to measure the value of each answer will be equal to below relationship:

$$
\text { (3) } \text { fitness }=\frac{1}{\sum_{1}^{N} E\left(p_{j}\right)}
$$

As a result, optimal answer is selected based on relationship (3) representing fitness functionality of algorithm selection with the lowest energy consumption in physical hosts.

\section{IV.IMPLEMENTATION AND ITS RESULTS}

In order to simulate cloud environment, cloudsim 3.0 simulator library designed and implemented based on Java and netbeans IDE 7.4 develop environment are being used.

A. surveying energy consumption level based on MAD and MMT algorithms

Surveying energy consumption level in GA and IWD algorithms in this section, some conditions will be considered so that overload detection in physical host and migrating VM selection would be respectively done based on MAD algorithm and MMT algorithm. Regarding achieved results of done surveys, it is obvious that in optimal answer; in the ten step of implementation; those five steps which have less processing load, using comparing algorithm intangibly consume less energy. In another words, utilizing IWD algorithm in this method offers better answers for data centers with high traffic which has excessive physical host load. The other problem pointed before about MMT algorithm is that this method would not be appropriate if we need a lot of memory free up to go out of overload state. While it works very well if a little free up would be sufficient. Anyway, what is ultimately considered is the average amount of energy consumption of different work conditions that in this case, Median Absolute Deviation algorithm is utilized to detect overload in physical host; 


\section{International Journal of Advanced Research in Computer and Communication Engineering}

Vol. 5, Issue 3, March 2016

and Minimum Migration method is used for VM selection. algorithm is equal to $43 \mathrm{~W} / \mathrm{H}$ which is an appropriate The average amount for 400 times of repetition for IWD answer in comparison with other discussed methods.

Table. 2. Algorithms' energy consumption amount using MAD and MMT algorithms

\begin{tabular}{|c|c|c|c|c|c|c|c|c|c|c|c|c|}
\hline & $\begin{array}{c}\text { Round } \\
1\end{array}$ & $\begin{array}{l}\text { Round } \\
2\end{array}$ & $\begin{array}{c}\text { Round } \\
3\end{array}$ & $\begin{array}{c}\text { Round } \\
4\end{array}$ & $\begin{array}{c}\text { Round } \\
5\end{array}$ & $\begin{array}{c}\text { Round } \\
6\end{array}$ & $\begin{array}{c}\text { Round } \\
7\end{array}$ & $\begin{array}{c}\text { Round } \\
8\end{array}$ & $\begin{array}{c}\text { Round } \\
9\end{array}$ & $\begin{array}{c}\text { Round } \\
10\end{array}$ & $\begin{array}{c}\text { AVG } \\
(\mathrm{KW} / \mathrm{H})\end{array}$ & $\begin{array}{l}\text { AVG } \\
(\mathrm{W} / \mathrm{H})\end{array}$ \\
\hline Proposed & 0.01 & 0.02 & 0.04 & 0.06 & 0.04 & 0.01 & 0.03 & 0.02 & 0.14 & 0.06 & 0.043 & 43 \\
\hline GA & 0.21 & 0.02 & 0.06 & 0.11 & 0.16 & 0.07 & 0.13 & 0.06 & 0.03 & 0.03 & 0.088 & 88 \\
\hline
\end{tabular}

Table 3. Algorithms' energy consumption amount using RS and IQR algorithms

\begin{tabular}{|c|c|c|c|c|c|c|c|c|c|c|c|c|}
\hline & $\begin{array}{c}\text { Round } \\
1\end{array}$ & $\begin{array}{c}\text { Round } \\
2\end{array}$ & $\begin{array}{c}\text { Round } \\
3\end{array}$ & $\begin{array}{c}\text { Round } \\
4\end{array}$ & $\begin{array}{c}\text { Round } \\
5\end{array}$ & $\begin{array}{c}\text { Round } \\
6\end{array}$ & $\begin{array}{c}\text { Round } \\
7\end{array}$ & $\begin{array}{c}\text { Round } \\
8\end{array}$ & $\begin{array}{c}\text { Round } \\
9\end{array}$ & $\begin{array}{c}\text { Round } \\
10\end{array}$ & $\operatorname{AVG}(\mathrm{KW} / \mathrm{H})$ & $\mathrm{AVG}(\mathrm{W} / \mathrm{H})$ \\
\hline Proposed & 0.06 & 0.01 & 0.01 & 0.03 & 0.04 & 0.01 & 0.02 & 0.1 & 0.06 & 0.11 & 0.045 & 45 \\
\hline GA & 0.12 & 0.08 & 0.09 & 0.1 & 0.1 & 0.02 & 0.04 & 0.02 & 0.03 & 0.03 & 0.063 & 63 \\
\hline
\end{tabular}

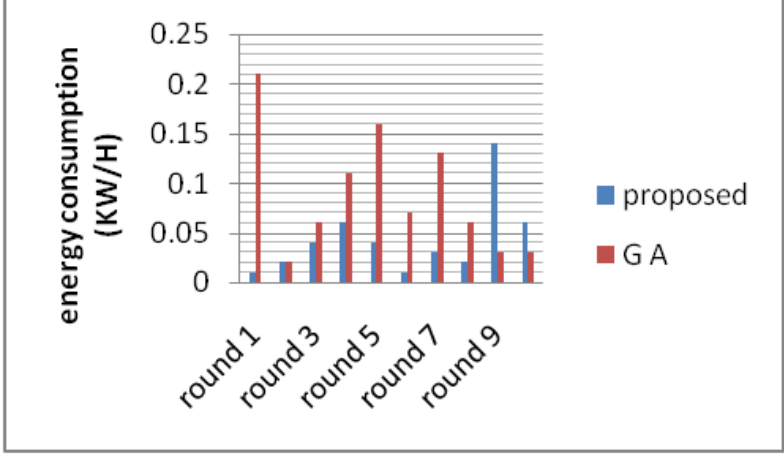

Diagram.1. Algorithms' energy consumption amount using MAD and MMT algorithms

B. surveying energy consumption amount based on $R S$ and IQR algorithms

Here in this case, for performance of GA and IWD algorithms, conditions are considered so that IQS and RS algorithms will be respectively used for overload detection in physical host and random selection of migrating VM. Just like previous sections, energy consumption amount is the matter of consideration.

As demonstrated in table and diagram (2), the amount of energy consumption has been surveyed using IQR algorithm for overload detection and RS algorithm for VM selection. When IQR and RS algorithms are used for selecting physical hosts and VMs, the average of 10 optimal answers of IWD algorithm show lower energy consumption comparing with the other three methods.

Surveying simulation results, it is found that in optimal answer of the ten-step implemented, again using compared algorithms consume intangibly less energy in 3 cases which in processing load of physical host is low. In another words, utilizing IWD algorithm in this method also offers a better answer for data centers with high traffic with excessive load of physical host. In this case, the average amount of energy consumption in different working conditions for IWD algorithm is 45 W/D. Although this amount of energy consumption has a better answer in comparison with other discussed methods when

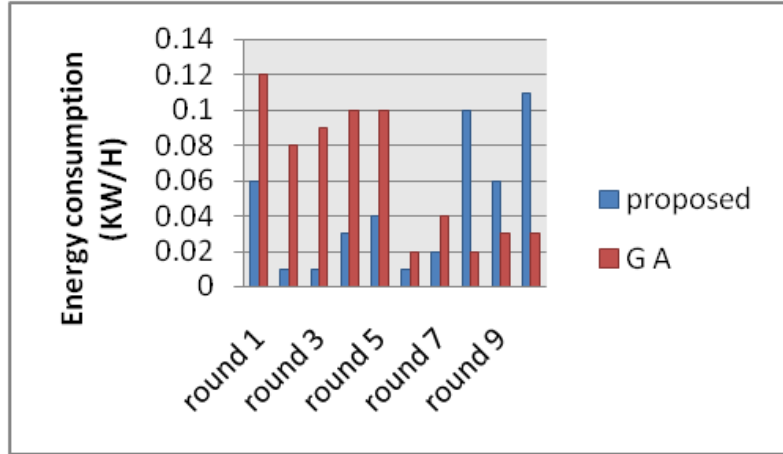

Diagram. 2. Algorithms' energy consumption amount using RS and IQR algorithms

using IQR and RS algorithms, but shows higher energy consumption comparing to the results of part 1-5. In another words, based on achieved results, Intelligent Drop algorithm consumes $2 \mathrm{~W} / \mathrm{H}$ less energy using MAD and MMT algorithms than using IQR and RS algorithms.

\section{CONCLUSION}

In proposed method which was based on Intelligent Water Drop Algorithm, optimal VM allocation to physical hosts of working resources became minimum and through this, the amount energy consumption in data centers was reduced. Important parameters of resource management in cloud network as the number, processing power, and memory capacity of physical and virtual machines have been mapped onto static and dynamic parameters which are speed and soil on IWDs in intelligent water drop algorithm. And the optimal answer is defined based on fitness functionality which is depending on the amount of energy consumption while being idle or in the processing peak and CPU efficiency amount in physical host.

Considering results of separate simulation of each one of IWD and GA algorithms and based on different policies of physical and virtual machines presented in implementation, the proposed method shows the least energy consumption as $43 \mathrm{~W} / \mathrm{H}$ as well as MAD algorithm as overload detection algorithm and MMT as VM 
International Journal of Advanced Research in Computer and Communication Engineering Vol. 5, Issue 3, March 2016

selection algorithm. Consequently considering comparisons done with other methods, IWD algorithm showed the most optimal answer for physical resource management through using MAD algorithms as overload detection algorithm and MMT algorithms as VM selection.

\section{REFERENCES}

[1] E. Kotsovinos, "Virtualization: Blessing or Curse? ACM Queue," (2011) IEEE Computer Magazine 7(6): 34-45.

[2] R. Kesavamoorthy, D. A. Shunmugam, L. T. Mariappan, "Solving traveling salesman problem by modified intelligent water drop algorithm," (2011) International Conference on Emerging Technology Trends (ICETT).

[3] A. Belglazov, J. Abawajy, R. Buyya," Energy-aware resource allocation heuristics for efficient management of data centers for cloud computing" Future Generation Computer Systems 28 (2012) 755-768.

[4] L. Zhu, Q. Li, L. He," Study on Cloud Computing Resource Scheduling Strategy Based on the Ant Colony Optimization Algorithm," IJCSI International Journal of Computer Science Issues,(2012) Vol. 9, Issue 5, No 2, ISSN (Online): 1694-0814.

[5] K. Lenin, M. Surya Kalavathi," An Intelligent Water Drop Algorithm for Solving Optimal Reactive Power Dispatch Problem" (2012) International Journal on Electrical Engineering and Informatics Volume 4, Number 3.

[6] D. PALANIKKUMAR, E. GOWSALYA, B. RITHU, p. ANBUSELVEN, "AN INTELLIGENT WATER DROPS ALGORITHM BASED SERVICE SELECTION AND COMPOSITION IN SERVICE ORIENTED ARCHITECTURE," Journal of Theoretical and Applied Information Technology,(2012) Vol. 39 No.1.

[7] JH. Lin, CW. Chou, CH. Yang, HI. Tsai, ” A chaotic Lévy flight bat algorithm for parameter estimation in nonlinear dynamic biological systems," (2014) Comput Inf Tech;2(2):56-63.

[8] O. A. Basem, A. Li-Pei Wong," A modified Intelligent Water Drops algorithm and its application to optimization problems, Expert Systems with Applications"(2014) Expert Systems with Applications 41, 6555-6569. 